Journal of Mathematics and Informatics

Vol. 6, 2016, 67-72

ISSN: 2349-0632 (P), 2349-0640 (online)

Published 27 December 2016

www. researchmathsci.org

DOI: http://dx.doi.org/10.22457/jmi.v6a8

Journal of

Mathematics and

Informatics

\title{
Reduction of Rough Set Based on Generalized Neighborhood System Operator
}

\author{
Fang-fang Zhao ${ }^{1}$ and Ling-qiang $\mathbf{L i}^{2}$ \\ Department of Mathematics, Liaocheng University \\ Liao'cheng - 252059, Liao'cheng, China \\ Email: ${ }^{1}$ 1016610977@qq.com, ${ }^{2}$ lilingqiang0614@126.com \\ ${ }^{2}$ Corresponding author
}

Received 17 December 2016; accepted 27 December 2016

\begin{abstract}
The theory of generalized neighborhood system-based approximation operators plays an important role in the theory of generalized rough sets since it includes both the neighborhood-based approximation operators and the covering-based approximation operators as its special circumstances. The theory of reduction is one of the most significant directions in rough sets. In this work, the reduction of rough set based on generalized neighborhood system operator is defined and discussed. In particular, the conditions for two generalized neighborhood system operator to generate the same lower or upper approximation are provided.
\end{abstract}

Keywords: rough set; neighborhood system; reduct; lower approximation operator; upper approximation operator

AMS Mathematics Subject Classification (2010): 03E02, 68T30

\section{Introduction}

Rough set theory, proposed by Pawlak [9], is an effective mathematical approach to deal with uncertainty, granularity and incompleteness of knowledge. It has been successfully applied to intelligent control, economic, biology, data mining, medical diagnosis, and elsewhere $[10,11,25,26]$.

The classical Pawlak's rough sets are based on partition or equivalent relation. This is too restrictive for many applications of Pawlak's rough sets. To address this problem, many extensions of a partition or equivalence relation have been proposed, such as tolerance relation [13], binary relations [17,22], similarity relations [14], coverings $[1,7,12,19,21,24]$, neighborhood systems [2,18].

The rough sets based on generalized neighborhood system is introduced by Lin -Yao [3,4], and then researched by Yao [18], Lin-Michael [2,8], Syau- Lin [15] and Zhang et al. [27]. It is observed in [27] that the generalized neighborhood system-based rough sets is more general than the neighborhood-based (binary relation-based) rough sets and covering-based rough sets. It is well known that reduction theory is an important part of rough set theory $[20,23]$. However, as to our knowledge, there is no work in the reduction of generalized neighborhood system-based rough sets. The main objective of this paper is 
Fang-fang Zhao and Ling-qiang Li

to serve such a purpose.

This paper is organized as follows. In Section 2, we recall some notions and results about generalized neighborhood system-based rough sets. In Section 3, we present the theory of reduction of rough sets based on generalized neighborhood system operator. In Section 4, we make a conclusion.

\section{Preliminaries}

In this section, we will introduce some basic concepts about generalized neighborhood system and rough sets based on generalized neighborhood system.

Definition 2.1. [2] Let $U$ be the universe of discourse, and $2^{U}$ denote the power set of $U$. Then a function $N: U \rightarrow 2^{2^{U}}$ is called a generalized neighborhood system operator on $U$. For any $x \in U, N(x)$ is non-empty. Usually, $N(x)$ is called generalized neighborhood system of $x$ and any $K \in N(x)$ is called neighborhood of $x$.

Definition 2.2. Let $N$ be a generalized neighborhood system operator of $U$ and $x \in U$. Then the set family

$$
M D_{N}(x)=\{K \in N(x) \mid \forall V \in N(x) \wedge V \subseteq K \Rightarrow K=V\}
$$

is called the minimal description of $N$ at $x$.

Definition 2.3. [5,6] Let $N$ be a generalized neighborhood system operator of $U$. For each subset $X$ of $U$, the lower and upper approximations of $X, \underline{N}$ and $\bar{N}$, respectively, are defined as follows:

$$
\underline{N}(X)=\{x \in U \mid \exists K \in N(x), K \subseteq X\}, \bar{N}(X)=\{x \in U \mid \forall K \in N(x), K \cap X \neq \varnothing\} .
$$

\section{Reduction of rough sets based on generalized neighborhood system}

In this section, we shall present the theory of reduction of rough sets based on generalized neighborhood system.

Definition 3.1. Let $N$ be a generalized neighborhood system operator of a universe $U$ and $x \in U$.

(1) For any $K \in N(x)$, we say $K$ is a reducible element of $N$ at point $x$ if there exists an $V \in N(x)$ such that $V \subset K$ (i.e., $V \subseteq K$ and $V \neq K$ ) otherwise $K$ is an irreducible element of $N$ at point $x$.

(2) If for any $K \in N(x), K$ is irreducible element of $N$ at point $x$, then we say $N$ is irreducible at point $x$, otherwise $N$ is reducible at point $x$.

Let $N$ be a generalized neighborhood system operator of a universe $U$. For any reducible element $K$ of $N$ at point $x$, we define a operator $N_{K}: U \rightarrow 2^{2^{U}}$ as

$$
N_{K}(z)=\left\{\begin{array}{cc}
N(z)-K, & z=x ; \\
N(z), & \text { others. }
\end{array}\right.
$$

It is easy to observe that the family $N_{K}(x)=N(x)-K$ is still non-empty since $K$ is reducible element of $N$ at point $x$. This shows that $N_{K}$ is also a generalized neighborhood system operator of the universe $U$. 
Reduction of Rough Set Based on Generalized Neighborhood System Operators

Proposition 3.1. Let $N$ be a generalized neighborhood system operator of a universe $U$ and $K$ be a reducible element of $N$ at point $x$. Then for any $K_{1} \in N_{K}(x), K_{1}$ is a reducible element of $N$ at point $x$ if and only if it is a reducible element of $N_{K}$ at point $x$.

Proof. $(\Leftarrow)$ It is obviously since $N_{K}(x) \subseteq N(x)$.

$\Leftrightarrow$ Let $K_{1}$ be a reducible element of $N$ at point $x$. Then there exists an $M \in N(x)$ such that $M \subset K_{1}$. If $M \neq K$ then $M \in N_{K}(x)$ and it follows that $K_{1}$ is a reducible element of $N_{K}$ at point $x$. If $M=K$, from that $K$ is a reducible element of $N$ at point $x$, there exists an $H \in N(x)$ such that $H \subset K=M \subset K_{1}$. Obviously, $H \in N_{K}(x)$, it follows that $K_{1}$ is a reducible element of $N_{K}$ at point $x$.

From Proposition 3.1 we observe easily that deleting a reducible element in a neighborhood system will not generate any new reducible elements or make other originally reducible element become irreducible elements of the new neighborhood system. Thus we can get the reduction of a neighborhood system of a universe $U$ by deleting all reducible elements at each point in the same time or by deleting one reducible element at each point in a step. The remainder still consists of a neighborhood system of the universe $U$, and it is irreducible. Thus we give the definition of neighborhood system reduction as follows:

Definition 3.2. Let $N$ be a generalized neighborhood system operator of a universe $U$. The generalized neighborhood system operator generated by deleting all reducible elements at each point, is called the reduct of $N$, and denoted by $\operatorname{reduct}(N)$.

Lemma 3.1. Let $N$ be a generalized neighborhood system operator of a universe $U$ and $x \in U$. Then $K$ is a reducible element of $N$ at point $x$ if and only if $K \notin M D_{N}(x)$.

Proof. $(\Rightarrow)$ Let $K$ be a reducible element of $N$ at point $x$, then there exists an $V \in N(x)$ such that $V \subset K$, by the definition 2.2 , we have $K \notin M D_{N}(x)$.

$(\Leftarrow)$ Let $K \in N(x)$ but $K \notin M D_{N}(x)$, then by the definition 2.2, there exists an $S \in N(x)$ such that $S \subset K$, hence $K$ is a reducible element of $N$ at point $x$.

By Lemma 3.1 and Definition 3.2 we get the following theorem.

Theorem 3.1. Let $N$ be a generalized neighborhood system operator of a universe $U$. Then $N$ and $\operatorname{reduct}(N)$ have the same minimal description at all $x \in U$.

\subsection{For lower approximation operator}

Lemma 3.1.1. Let $N$ be a generalized neighborhood system operator of a universe $U$ and $K$ be a reducible element of $N$ at point $x$. Then $N$ and $N_{K}$ generate the same lower approximation operator. That is, $\underline{N}(X)=\underline{N_{K}}(X)$ for all $X \subseteq U$.

Proof. Let $X \subseteq U$. Obviously, $\underline{N_{K}}(X) \subseteq \underline{N}(X)$ by $\underline{N_{K}}(x) \subseteq \underline{N}(x)$ for all $x \in X$. Conversely, let $x \in \underline{N}(X)$. By definition 2.3, there exists an $M \in N(x)$ such that $M \subseteq X$. If $M \neq K$ then we have $M \in N_{K}(x)$, and hence $x \in \underline{N_{K}}(X)$. If $M=K$, since $K$ is a reducible element 
Fang-fang Zhao and Ling-qiang Li

of $N$ at point $x$, then there exists an $V \in N(x)$ such that $V \subset K=M$, which means $V \in N_{K}(x)$, and so $x \in \underline{N_{K}}(X)$. Thus $\underline{N_{K}}(x) \supseteq \underline{N}(x)$.

By Lemma 3.1.1, we get the following corollary.

Corollary 3.1.1. Let $N$ be a generalized neighborhood system operator of a universe $U$. Then $\quad N$ and $\operatorname{reduct}(N)$ generate the same lower approximation operator.

Proposition 3.1.1. Let $N_{1}, N_{2}$ be two irreducibly generalized neighborhood system operators of a universe $U$ generating the same lower approximation operator. Then $N_{1}=N_{2}$.

Proof. For any $K \in N_{1}(x)$, by definition 2.3, we have $x \in \underline{N_{1}}(K)=\underline{N_{2}}(K)$, then there exists an $K^{\prime} \in N_{2}(x)$ such that $K^{\prime} \subseteq K$. Similar to the above proof, there exists an $K^{\prime \prime} \in N_{1}(x)$ such that $K^{\prime \prime} \subseteq K^{\prime} \subseteq K$. Since $N_{1}$ is irreducible, then we get $K^{\prime \prime}=K$, and then $K=K^{\prime} \in N_{2}(x)$. It follows immediately that $K \in N_{1} \Leftrightarrow K \in N_{2}$. Hence $N_{1}=N_{2}$.

By Corollary 3.1.1 and Proposition 3.1.1, we get the following theorem.

Theorem 3.1.1. Let $N_{1}, N_{2}$ be two generalized neighborhood system operators of a universe $U$. Then $N_{1}, N_{2}$ generate the same lower approximation operator if and only if $\operatorname{reduct}\left(N_{1}\right)=\operatorname{reduct}\left(N_{2}\right)$.

\subsection{For upper approximation operator}

By dualizing the results on lower approximation operator we get the following results on upper approximation operator. We omit the similar proofs.

Lemma 3.2.1. Let $N$ be a generalized neighborhood system operator of a universe $U$ and $K$ be a reducible element of $N$ at point $x$. Then $N$ and $N_{K}$ generate the same upper approximation operator. That is, $\bar{N}=\overline{N_{K}}$ for all $X \subseteq U$.

Corollary 3.2.1. Let $N$ be a generalized neighborhood system operator of a universe $U$. Then $N$ and $\operatorname{reduct}(N)$ generate the same upper approximation operator.

By Corollary 3.1.1 and 3.2.1, we get the following corollary.

Corollary 3.2.2. Let $N$ be a generalized neighborhood system operator of a universe $U$. Then $N$ and $\operatorname{reduct}(N)$ generate the same upper and lower approximation operators.

Proposition 3.2.1. Let $N_{1}, N_{2}$ be two irreducibly generalized neighborhood system operators of a universe $U$ generating the same upper approximation operator. Then $N_{1}=N_{2}$.

By Corollary 3.2.1 and Proposition 3.2.1, we get the following theorem. 
Reduction of Rough Set Based on Generalized Neighborhood System Operators

Theorem 3.2.1. Let $N_{1}, N_{2}$ be two generalized neighborhood system operators of a universe $U$. Then $N_{1}, N_{2}$ generate the same upper approximation operator if and only if $\operatorname{reduct}\left(N_{1}\right)=\operatorname{reduct}\left(N_{2}\right)$.

From Theorem 3.1.1 and 3.2.1, we get the following corollary.

Corollary 3.2.3. Let $N_{1}, N_{2}$ be two generalized neighborhood system operators of a universe $U$. Then $N_{1}, N_{2}$ generate the same upper approximation operator if and only if they generate the same lower approximation operator.

\section{Conclusions}

In this paper, we discuss the theory of reduction of rough set based on generalized neighborhood system operator, and present the conditions for two generalized neighborhood system operator to generate the same generalized neighborhood systembased lower or upper approximation operator.

\section{Acknowledgements}

This work is supported by National Natural Science Foundation of China (11501278) and Shandong Provincial Natural Science Foundation, China (ZR2013AQ011, ZR2014AQ011).

\section{REFERENCES}

1. Z.Bonikowski, E.Bryniarski and U.W.Skardowska, Extensions and intentions in the rough set theory, Information Sciences, 107 (1998) 149-167.

2. T.Y.Lin, Neighborhood systems-application to qualitative fuzzy and rough sets, Advances in Machine Intelligence and Soft Computing IV, (1997) 132--155.

3. T.Y.Lin, Granular computing on binary relations I: Data mining and neighborhood systems, in: A.Skowron, L.Polkowski (Eds.), Rough Sets and Knowledge Discovery, Physica-Verlag, 1998, pp. 107-121.

4. T.Y.Lin, Q.Liu, K.J.Huang and W.Chen, Rough sets, neighborhood systems and approximation, Methodologies for Intelligent System, 1990, pp. 130-141.

5. T.Y.Lin and Y.Y.Yao, Mining soft rules using rough sets and neighborhoods. in: Proceedings of the Symposium on Modelling, Analysis and Simulation, Computational Engineering in Systems Applications (CESA 96), IMASCS Multiconference, Lille, France, 1996.

6. T.Y.Lin and Y.R.Syau, Unifying Variable Precision and Classical Rough Sets: Granular Approach, Rough Sets and Intelligent Systems-Professor Zdzislaw Pawlak in Memorian (Vol.II), Intelligent Systems Reference Library, 42 (2013) 287-304.

7. T.J.Li, Rough approximation operators in covering approximation spaces, RSCTC 2006, LNCS, 4259 (2006) 174-182.

8. J.B.Michael and T.Y.Lin, Neighborhoods, Rough sets, and Query Relaxation in Cooperative Answering, Rough Sets and Data Mining: Analysis of Imprecise Data, Kluwer Academic Publisher, 1997, pp. 229-238.

9. Z.Pawlak, Rough sets, International Journal of Computer and Information Sciences, 11 (1982) 341-356.

10. Z. Pawlak, Rough Sets: Theoretical Aspects of Reasoning about Data, Kluwer Academic Publishers, Boston, 1991. 
Fang-fang Zhao and Ling-qiang Li

11. L.Polkowski and A.Skowron (Eds.), Rough Sets in Knowledge Discovery, PhysicVerlag, Heidelberg, 1998.

12. K.Qin, Y.Gao and Z.Pei, On covering rough sets, RSKT, LNCS, 2007, pp. 34-41.

13. A.Skowron and J.Stepaniuk, Tolerance approximation spaces, Fundamenta Informaticae, 27 (1996) 245-253.

14. R.Slowinski and D.Vanderpooten, A generalized definition of rough approximations based on similarity, IEEE Trans. Data Knowledge Eng, 12 (2000) 331-336.

15. Y.R.Syau and E.B.Lin, Neighborhood systems and covering approximate spaces, Knowledge-Based Systems, 66 (2014) 61-67.

16. Y.Y.Yao, Constructive and algehraic methods of theory of rough sets, Information Sciences, 109 (1998) 21-47.

17. Y.Y.Yao, Relational interpretations of neighborhood operators and rough set approximation operators, Information Sciences, 111 (1998) 239-259.

18. Y.Y.Yao, Neighborhood systems and approximate retrieval, Information Sciences, 176 (2006) 3431-3452.

19. Y.Y.Yao and B.X.Yao, Covering based rough set approximations, Information Sciences, 200 (2012) 91-107.

20. T.Yang and Q.G.Li, Reduction about approximation spaces of covering generalized rough sets, International Journal of Approximation Reasoning, 51 (2010) 335-345.

21. W.Zhu, Topological approaches to covering rough sets, Information Sciences, 177 (2007) 1499-1508.

22. W.Zhu, Relationship between generalized rough sets based on binary relation and covering, Information Sciences, 179 (2009) 210-225.

23. W.Zhu and F.Y. Wang, Reduction and axiomization of covering generalized rough sets, Information Sciences, 152 (2003) 217-230.

24. W.Zhu and F.Y.Wang, Relationships among three types of covering rough sets, IEEE GrC, 2006, pp. 43-48.

25. W.Zhu and F.Y.Wang, Covering based granular computing for conflict analysis, IEEE ISI, 3975 (2006) 566-571.

26. N.Zhong, Y.Yao and M.Ohshima, Peculiarity oriented multidatabase mining, IEEE Transactions on Knowledge and Data Engineering, 15 (2003) 952-960.

27. Y.L.Zhang, C.Q.Li , M.L.Lin and Y.J.Lin, Relationships between generalized rough sets based on covering and reflexive neighborhood system, Information Sciences, 319 (2015) 56-67. 